\title{
Motorsafe: An Android Application for Motorcyclists Using Decision Tree Algorithm
}

\author{
https://doi.org/10.3991/ijim.v14i02.10742 \\ Thang Van Nguyen \\ ThuyLoi University, Hanoi, Vietnam \\ Van Dung Nguyen \\ Nguyen Tat Thanh University, Ho Chi Minh City, Vietnam \\ Thi-Thu Nguyen \\ Hanoi University of Industry, Hanoi, Vietnam \\ Phung Cong Phi Khanh \\ VNU University of Engineering and Technology, Hanoi, Vietnam \\ Tien-Anh Nguyen \\ Le Quy Don Technical University, Hanoi, Vietnam \\ Duc-Tan Tran $(\bowtie)$ \\ Phenikaa University, Hanoi, Vietnam \\ Phenikaa Research and Technology Institute (PRATI), Hanoi Vietnam \\ tan.tranducephenikaa-uni.edu.vn
}

\begin{abstract}
In Vietnam, a ban on mobile phone use while driving has been in effect for a long time because this habit is highly dangerous to both drivers and other road users, causing distracted driving and resulting in multiple crashes. MotorSafe is a novel mobile application that has been launched as a solution for dealing with mobile phone usage while operating a vehicle. Based on the data obtained from the accelerometer sensor on the smartphone, the decision tree algorithm was made use of, which enabled the device to recognize the app user's status. Some necessary tasks then followed to help the biker focus on operating his vehicle instead of displaying unnecessary behaviors.
\end{abstract}

Keywords-Android, Acceleration, Driving, Decision tree, Algorithm, Classification

\section{Introduction}

According to the Traffic Police Department under the Ministry of Public Security, there are about 60 million motorbikes in circulation in Vietnam, accounting for $93 \%$ of the total vehicle numbers. Therefore, motorcyclists account for a high proportion (approximately 59\%) of the road traffic collisions in the country, causing ten thousand 
deaths and injuries each year and tragically many of whom are teenagers. Bikers are reported to frequently use mobile phones, or smartphones given today's phone technology while controlling their vehicle, leading to biker distraction as well as their reduced ability to observe and control their speed. Unexpected events hence might occur and in most cases these could have catastrophic consequences.

In recent years, in order to reduce the number of traffic accidents and fatalities as well as to protect drivers, several countermeasures have been developed by researchers and companies around the world. A variety of mobile phone apps have been designed to act as voluntary 'workload managers', which work to prevent distracted driving due to mobile phone use, but most of them are for cars [3-6]. The pioneer in developing apps to protect two wheeler riders, the app S-Bike, which will come preinstalled on all 4G-enabled Samsung Galaxy J Series devices in the future, is designed to enable responsible and safe riding. S-Bike can filter incoming calls but also allow receiving the calls from important people after the motorcyclist brings his bike to a halt [2]. However, S-Bike has several major limitations in that it cannot automatically switch between S-Bike mode and normal mode and the biker needs to do this manually. This is extremely inconvenient and problematic because the smartphone is still on S-bike mode after the biker halts, thus he or she might miss certain important incoming calls if he or she forgets or fails to switch the phone back to the normal mode. Furthermore, this application is only available on the costly products of Samsung, which means all other Android cell phones cannot have this app installed.

Therefore, in this study, we propose an effective application that can be installed on all Android smartphones to reduce crash rates associated with mobile phone use while driving; it is named "Motorsafe". The application will automatically recognize exactly the states of the user. For example, while the biker is detected "On vehicle", the phone will automatically change to the silent mode and all incoming calls from unimportant or unknown people will be rejected. When the biker stops the bike, the phone will also automatically return to the normal mode.

In the first version, this software is developed using Google Activity Recognition API (GAR_API) with its machine learning classifiers to recognize user's activities. This algorithm has an advantage in that it has available, well-studied functions which can be applied immediately with a high accuracy. However, this advantage is also a drawback of that algorithm as the available functions should have parameters which cannot be adjusted and exploited other features when using this algorithm. (I really don't get what you mean here). In addition, the GAR_API algorithm is extremely complex and ill-suited to the lower configuration phones.

In this study, we developed a simpler algorithm, the Decision Tree Algorithm, which can be used on a number of Android cellphones with different configurations including the budget smart phones. Therefore, our application can approach a wider range of users. This algorithm will detect exact behaviors of the users: "On vehicle" or "Not on vehicle" by analyzing the information obtained from built-in accelerometer sensors on Android smartphones. After that, a number of tasks will be performed based on the detected status: 
- Automatically detecting the status of users and changing the phone to silent mode when the user is riding a two-wheeler, and switching the phone back to normal mode when the user brings their motorbike to a halt.

- Rejecting all unimportant and unknown incoming calls and automatically sending a message to inform the caller of the user's "On vehicle" state.

- Allowing receiving incoming calls from important people (VIP) regardless of whether the user is on the vehicle or not. The list of VIPs can be erased, modified or replaced.

- Handling emergency calls (not in the VIPs list), calls which are identified as continuous call frequency ( 3 calls in 3-5 minutes), in the same vein as calls from the VIPs list.

- Warning traffic participants if they are speeding.

- Detecting an accident and automatically sending a message notification together with the exact location of the crash to the biker's relatives or to hotline of the nearest hospitals.

Our application is available on the Google Play [17].

\section{$2 \quad$ Material and Method}

\subsection{Data collection and feature extraction}

In this paper, the data was collected directly from the accelerometer sensor, then these data were processed to determine the user's "On Driving" behavior through the motions of the vehicle.

The raw data stream from the accelerometers is the acceleration of each axis $\mathrm{X}, \mathrm{Y}$, $\mathrm{Z}$ in the units of $\mathrm{g}$-force. Most of the existing accelerometers provide a user interface to configure the sampling frequency so that the user could choose the most suitable sampling rate through experiments. After collecting the raw data from the accelerometer sensor, the next step is to pre-process it before performing any further statistical computations. One purpose of the data pre-processing is to reduce the noise from the sensors.

To classify the users' activities, we used two different components of the raw acceleration data: static and dynamic. Firstly, the static component of the acceleration was caused by the orientation of the sensor which is proportional to the gravity and this static component can be obtained after passing low pass filter (LPF). Secondly, the dynamic component of the acceleration was caused directly by the movement of the accelerometer in the smartphone. The Dynamic Body Acceleration can be calculated by subtracting the static component of the acceleration from the corresponding raw accelerometer. Overall dynamic body acceleration (ODBA) and its vectorial variation (VeDBA) represented an aggregated acceleration used to detect different states [7]. Therefore, the dynamic body acceleration is a good candidate to discriminate between behaviors with high dynamic movements (such as walking or running) from behaviors and with low dynamic movements (such as on vehicles or on the table). 


$$
D B A_{i t}=A_{i t}=\left|x[i]_{t}-y[i]_{t}\right|,
$$

where $\mathrm{i}=\mathrm{X}, \mathrm{Y}, \mathrm{Z}$

Ai: the dynamic acceleration data

$y[i]$ : the filtering acceleration data

$\mathrm{x}[\mathrm{i}]$ : the raw acceleration data

Then, the obtained values for DBA are used to calculate the overall dynamic body acceleration (ODBA) and the vectorial dynamic body acceleration (VeDBA)

$$
\begin{gathered}
O D B A=\left|A_{X}+A_{Y}+A_{Z}\right| \\
V e D B A=\sqrt{A_{X}^{2}+A_{Y}^{2}+A_{Z}^{2}}
\end{gathered}
$$

\subsection{Data classification}

The decision tree is one of the most popular machine learning algorithms used for both classification and regression problems [12][13]. The general motivation behind the deployment of the Decision Tree is to create a training model which can be used to predict the class or value of the target variables by learning the decision rules inferred from prior data (training data).

In the field of machine learning, the decision tree is a type of predictive models; it means a mapping from observations of a thing / phenomenon to the conclusion of a target value of a thing / phenomenon. Each internal node corresponds to a variable, the connection between it and its child node corresponds to a specific value for that variable. Each leaf node represents the predicted value of the variable target which depends on the values of the input that the variables go through, a path from the root node to the leaf node [8].

There are many specific decision-tree algorithms such as ID3, C4.5, CART, and CHAID. Since the ROC analysis is increasingly recognized as an important tool for evaluation and comparison of classifiers, we decided to use receiver operating curve (ROC) to define the threshold with aim of improving the quality of the decision tree algorithm in this study. In our research, we took into consideration two main modes: On Vehicle and Not-on Vehicle. For the Not-on Vehicle mode, we divided it into low dynamic activities (denoted by Not-on Vehicle (1)) and high dynamic ones (denoted by Not-on Vehicle (2)). The proposed flowchart for classification is shown in Fig. 1. 


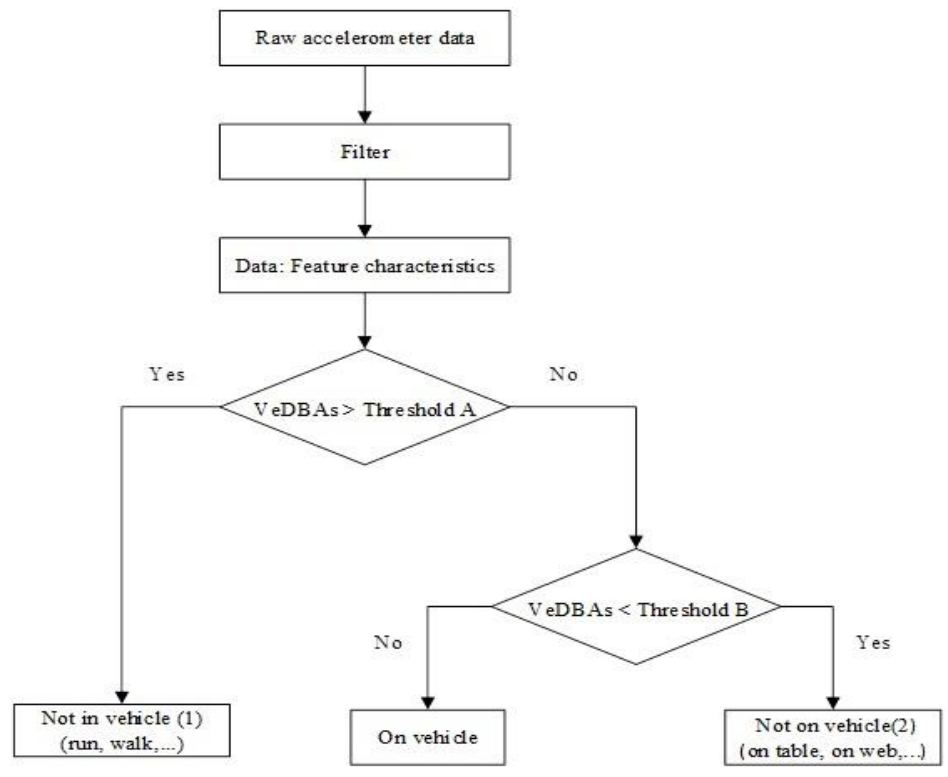

Fig. 1. The decision tree algorithm flowchart

The feature VeDBAs was then brought to the flow (as shown in Figure 2). VeDBAs values were compared with a predefined threshold A to differentiate between cases with high and low dynamic activities. In case of high dynamic activities (such as walking and running), the output was marked by "Not on vehicle (1)". In case of low dynamic activity, the system continued to compare the VeDBAs with threshold B in order to classify the data under two classes "On vehicle" and "Not on vehicle (2)".

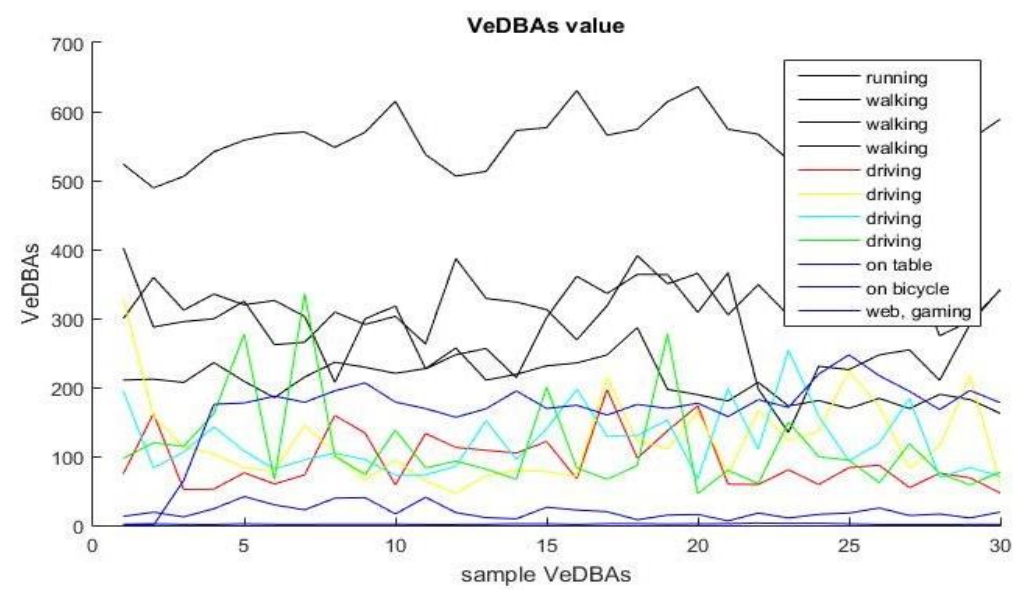

Fig. 2. Sequence of VeDBAs after preprocessing 


\section{Results and Discussion}

Firstly, the raw acceleration data was acquired from the acceleration sensor with a sampling frequency of $50 \mathrm{~Hz}$. A data frame with two-second episodes in real time (VeDBA) was then processed. As a consequence, each data frame consisted of 100 samples and the algorithm subsequently converted this frame into 1 element (i.e. each element was a sum of 100 continuous samples). After that, we used the confusion matrix in order to evaluate the performance of the detected algorithm [10-11]. TP (true positive) and TN (true negative) are correctly predicted cases, while FP (false positive) and FN (false negative) are the wrong predicted and unexpected cases. FN is equivalent to type-I error (rejecting the event as a positive event and assigning negative event) and FP is equivalent to type II error (accepting an event as a positive event when the event is negative in reality). The best prediction method that can produce a point in the upper left corner of the ROC space. For example, 100\% of true positive cases were found and $0 \%$ of false positive ones at all. The predicted random-line resulted in a straight line which formed a 45-degree angle with the horizontal axis from the bottom left to the upper right. The model could predict better recognition than random predictions when it had ROC on the left side of the random line. The best value of the cut point of Positive and Negative classification was the asymptotic point of the line which was parallel to the random line with the ROC curve. Finally, the optimal values of the thresholds were identified as:

- -Threshold A: $169 \mathrm{~m} / \mathrm{s} 2$

- Threshold B: $45 \mathrm{~m} / \mathrm{s} 2$

The thresholds are used to classify three states as shown in Fig.1. In this paper, the performance of the system was measured by calculating the precision and specificity using the following equations

$$
\begin{aligned}
& \text { Precision: } \begin{array}{l}
\text { pre }=\frac{T P}{T P+F P} \\
\text { Specificity: }
\end{array} \text { spe }=\frac{T N}{T N+F P}
\end{aligned}
$$

Twelve volunteers were randomly selected from different groups of students at VNU University of Engineering and Technology - Hanoi, Vietnam. The raw acceleration data was acquired from the acceleration sensor with the rate of 50 samples per second. Each state was recorded in approximately 30 minutes $(50 \%$ for training and $50 \%$ for testing) by each volunteer. Experimental data was then analyzed and the average performance of system is shown in Table 1. Our findings revealed the simplicity and remarkable effectiveness of the decision tree algorithm. 
Table 1. Average Performance (mean \pm standard deviation) of our software

\begin{tabular}{|l|c|c|}
\hline \multicolumn{1}{|c|}{ Status } & Precision (\%) & Specificity (\%) \\
\hline Not on vehicle (1) (on table, web surfing,...) & $100 \pm 0$ & $100 \pm 0$ \\
\hline On vehicle & $98.11 \pm 3.02$ & $96.77 \pm 1.12$ \\
\hline Not on vehicle(2) (running, walking,...) & $88.06 \pm 1.05$ & $91.11 \pm 2.24$ \\
\hline Overall & $95.39 \pm 1.39$ & $95.96 \pm 3.12$ \\
\hline
\end{tabular}

The findings indicated that the specificity and precision of the system were considerably high. Specifically, the precision of "On vehicle" was particularly high because there were only a few instances, which were observed "Not on vehicle" in reality but were predicted "On vehicle" state by algorithm. It demonstrated that our algorithm is an optimal solution.

After being installed on the user's smartphone, the application needs to be activated only once. From then on, the software will automatically detect the behavior of the users to specify whether they are driving or not driving. If the behavior "On Vehicle" is detected or when the user starts driving, the app will automatically change the phone from normal mode to silent mode and switch the phone back to normal mode when the user stops their motorbike.

The Motorsafe software has multiple important features as shown in the Introduction section. Figure 3 shows sample setting menus for some of the above-mentioned features. Our application is available on the Google Play at [17].

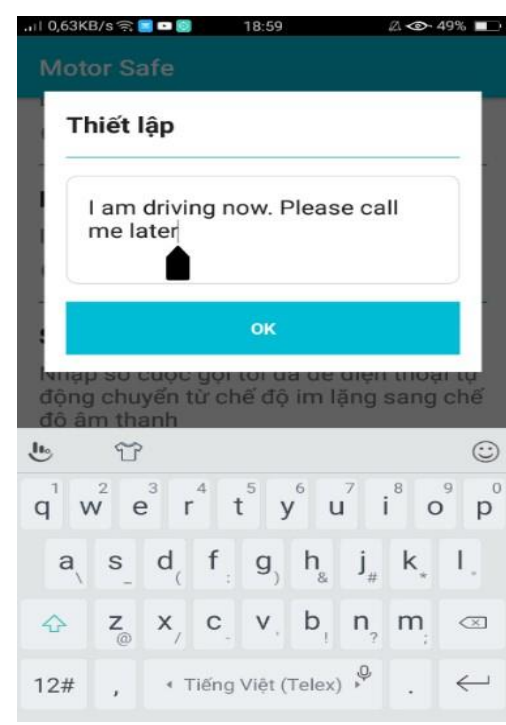

(a)

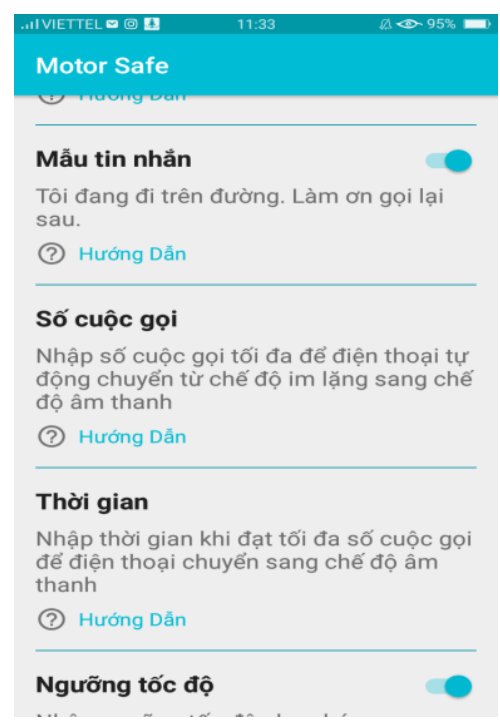

(b) 


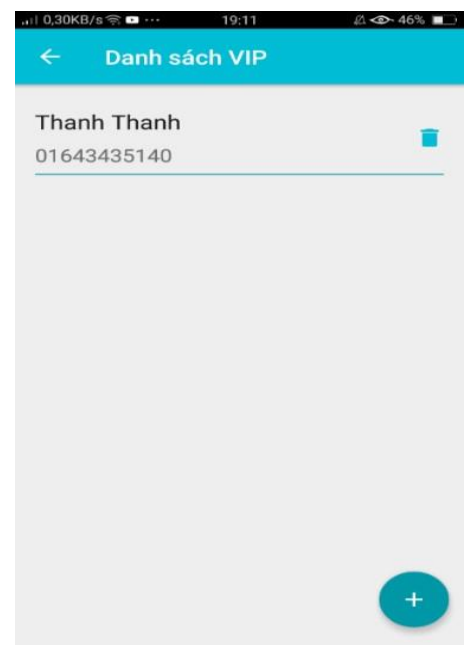

(c)

Fig. 3. Setting the content of the pre-prepared message or quick responses to send to the caller (a), Setting the Emergency call (b), Setting the VIPs list (c)

\section{Conclusion}

In this paper, we have successfully developed a "full-stack" Android application, the Motorsafe, with highly practical functions which has been widely used. Firstly, the decision tree algorithm was utilized to classify human behavior based on analyses of data obtained from the accelerometer in the smartphones. The reason for using the decision tree algorithm was that our software could be installed on low-cost smartphones, thereby reaching more users. Secondly, we have developed certain services after identifying the behavior "On Vehicle" of the users. There are many kinds of Android phones together with various built-in sensors, which are available in the market and with which this application is compatible. Motorsafe is a good example of cutting-edge mobile technologies which can be implemented in almost every aspect of our lives [13-16]. Currently, electroencephalogram-based brain computer studies are very promising and have been used for control applications [18-21]. With the strong development of mobile technology, the electroencephalogram-based brain - mobile phone interface will be an interesting research topic.

\section{Acknowledgement}

This work was supported by The Phenikaa University Foundation for Science and Technology Development. 


\section{$6 \quad$ References}

[1] $\mathrm{Vu}$ Anh Tuan, "Motorcycle Accidents in Vietnam", A Roundtable on safety of twowheeled vehicles, Geneva, 23-26 March 2015.

[2] Car \& Bike, "Samsung Launches New Smartphone With S Bike Mode", https://auto.ndtv. com/news/samsung-launches-new-smartphone-with-s-bike-mode-1292621, Access 22, April 2019.

[3] Su, X., Tong, H., \& Ji, P. (2014). “Activity recognition with smartphone sensors”, Tsinghua science and technology, 19(3), 235-249. https://doi.org/10.1109/tst.2014.6838194

[4] Wang, S., Chen, C., \& Ma, J. (2010, April). "Accelerometer based transportation mode recognition on mobile phones", 2010 Asia-Pacific Conference on Wearable Computing Systems, pp. 44-46. https://doi.org/10.1109/apwcs.2010.18

[5] Siirtola, P., \& Röning, J. (2012). "Recognizing human activities user-independently on smartphones based on accelerometer data", International Journal of Interactive Multimedia and Artificial Intelligence, Vol. 1(5), pp. 38-45. https://doi.org/10.9781/ijimai.2012.155

[6] Reddy, S., Mun, M., Burke, J., Estrin, D., Hansen, M., \& Srivastava, M. (2010). "Using mobile phones to determine transportation modes", ACM Transactions on Sensor Networks (TOSN), 6(2), 13. https://doi.org/10.1145/1689239.1689243

[7] Qasem, L., Cardew, A., Wilson, A., Griffiths, I., Halsey, L. G., Shepard, E. L., Wilson, R. (2012). "Tri-axial dynamic acceleration as a proxy for animal energy expenditure; should we be summing values or calculating the vector?", PloS one, 7(2), e31187. https://doi.org/ $\underline{10.1371 / \text { journal.pone. } 0031187}$

[8] Harrington, P. (2012). Machine learning in action (Vol. 5). Greenwich: Manning.

[9] Ali, H. M., \& Alwan, Z. S. (2017). Car accident detection and notification system using smartphone. Saarbrucken: LAP LAMBERT Academic Publishing.

[10] Fawcett, T. (2006). "An introduction to ROC analysis", Pattern recognition letters, 27(8), 861-874. https://doi.org/10.1016/j.patrec.2005.10.010

[11] Sing, T., Sander, O., Beerenwinkel, N., \& Lengauer, T. (2005). "ROCR: visualizing classifier performance in R", Bioinformatics, 21(20), 3940-3941. https://doi.org/10.1093/bioinf ormatics/bti623

[12] Phung Cong Phi, K., Nguyen Thi, K., Nguyen Dinh, C., Tran Duc, N., \& Tran Duc, T. (2019). Classification of cow's behaviors based on 3-DoF accelerations from cow's movements. International Journal of Electrical and Computer Engineering, Vol. 9(3), pp. 1656-1662. https://doi.org/10.11591/ijece.v9i3.pp1656-1662

[13] Hoang, Q. T., Phung, C. P. K., Bui, T. N., Chu, T. P. D., \& Tran, D. T. (2018). Cow Behavior Monitoring Using a Multidimensional Acceleration Sensor and Multiclass SVM. International Journal of Machine Learning and Networked Collaborative Engineering, Vol. 2(3), pp. 110-118. https://doi.org/10.30991/ijmlnce.2018v02i03.003

[14] Saare, M. A., Hussain, A., \& Yue, W. S. (2019). Investigating the Effectiveness of Mobile Peer Support to Enhance the Quality of Life of Older Adults: A Systematic Literature Review. International Journal of Interactive Mobile Technologies, Vol. 13(4), pp. 130-139. https://doi.org/10.3991/ijim.v13i04.10525

[15] Rojas-Osorio, M., \& Alvarez-Risco, A. (2019). Intention to Use Smartphones among Peruvian University Students. International Journal of Interactive Mobile Technologies, Vol. 13(3), pp. 40-52. https://doi.org/10.3991/ijim.v13i03.9356

[16] Alotaibi, M., Albalawi, M., \& Alwakeel, L. (2018). A Smart Mobile Pregnancy Management and Awareness System for Saudi Arabia. International Journal of Interactive Mobile Technologies (iJIM), Vol. 12(5), pp. 112-125. https://doi.org/10.3991/ijim.v12i5.9005 
Motor Safe, Google Play, https://play.google.com/store/apps/details?id=com.uetsign.motor $\underline{\text { safe\&hl}=e n}$

[17] Katona, J., \& Kovari, A. (2016). A Brain-Computer Interface Project Applied in Computer Engineering. IEEE Transactions on Education, 59(4), 319-326. https://doi.org/10.1109/te. $\underline{2016.2558163}$

[18] Katona, J., \& Kovari, A. (2018). The Evaluation of BCI and PEBL-based Attention Tests. Acta Polytechnica Hungarica, 15(3), 225-249. https://doi.org/10.12700/aph.15.3.2018.3.13

[19] Katona, J., \& Kovari, A. (2018). Examining the Learning Efficiency by a Brain-Computer Interface System. Acta Polytechnica Hungarica, 15(3), 251-280. https://doi.org/10.12700/ aph.15.3.2018.3.14

[20] Katona, J., Peter, D., Ujbanyi, T., \& Kovari, A. (2014). Control of incoming calls by a windows phone based brain computer interface. 2014 IEEE 15th International Symposium on Computational Intelligence and Informatics (CINTI). https://doi.org/10.1109/cinti.2014. $\underline{7028661}$

\section{Authors}

Thang Van Nguyen was born in 1979. He received his B.Sc., degree in Electronics and Telecommunication at the Hanoi University of Transport and Communications, Hanoi, Vietnam, in 2002 and his M.Sc. degree in Information Engineering from Le Quy Don University, Hanoi, Vietnam, in 2007. He received his PhD. degree in Electronics and Telecommunication at VNU University of Engineering and Technology. Now, he is lecturer at ThuyLoi University. Until now, he is author and coauthor of some papers on MEMS based sensors and their application.

Van Dung Nguyen received the B.Eng. and M.Eng. degrees in telecommunications engineering from the Ho Chi Minh City University of Technology, Vietnam, in 2008 and 2012, respectively. He is currently pursuing the Ph.D. degree in computer science and engineering with Kyung Hee University. He was a Post-Doctoral Researcher with the ICNS Laboratory, Kyung Hee University. From 2009 to 2010, he was a Developer with TOSADENSHI, Vietnam. From 2010 to 2013, he joined Ton Duc Thang University, Vietnam, where he was a Lecturer of electronics and telecommunications. From 2013 to 2018, he received the scholarship for his graduate study from Kyung Hee University. His research interests include designing the MAC protocols in vehicular ad hoc network and WSN.

Thi-Thu Nguyen was born in Hanoi, Vietnam, in 1977. She received the B. Eng. degree from University of Transport and Communications Hanoi, Viet-nam, in 2000. She received his master of engineering (ME) in telecommunications engineering from University of Engineering and Technology, Hanoi, Vietnam, in 2005. She received her PhD. degree engineering in electronic engineering from the Le Quy Don Technical University, Hanoi, Vietnam in 2018. She is currenly working at Hanoi University of Industry. Her current research interests include space-time processing, spacetime coding and embedded systems.

Phung Cong Phi-Khanh received the B.S. degree in Technology Education, Hanoi National University of Education in 1998 and M.S. degree in Electronic and Communication from VNU University of Engineering and Technology (VNU-UET) 
in 2005. He is currently Phd. student at VNU-UET. His present research interests are in digital signal processing and embedded systems.

Tien Anh Nguyen received the BSc degree in Physics and MSc degree in Electronics-Telecommunication Technology from Vietnam National University, Hanoi (VNU) in 2003 and in 2007, respectively. He received the Dr.-Eng degree from the Department of Microsystems Engineering (IMTEK), University of Freiburg, Germany in 2015. His research interests include cell-based impedance biosensors, microfluidics, single cell analysis, and applied of sensors system. He has joined the Faculty of Physical-Chemical Engineering, Le Quy Don Technical University since 2016. His currently is the principal investigator of 1 Nafosted project and 1 ministry project.

Tran Duc-Tan is an Associate professor and Vice Dean of Faculty of Electrical and Electronic Engineering, Phenikaa University. He has published over 150 research papers. His publications received the "Best Paper Award" at the 9th International Conference on Multimedia and Ubiquitous Engineering (MUE-15), and International Conference on Green and Human Information Technology (ICGHIT-2015). He was the recipient of the award for the excellent young researcher from Vietnam National University in 2008, Hanoi and the third prize in the contest "Vietnamese Talents" in 2008. His main research interests include the representation, processing, analysis, and communication of information embedded in signals and datasets. He serves as a TP Co-chair, technical committee program member, track chair, session chair and reviewer of many international conferences and journals.

Article submitted 2019-04-26. Resubmitted 2019-11-27. Final acceptance 2019-11-27. Final version published as submitted by the authors. 